\title{
Cooperative Diversity with Multiple-Antenna Nodes in Fading Relay Channels
}

\author{
Hakam Muhaidat, Member, IEEE, and Murat Uysal, Senior Member, IEEE
}

\begin{abstract}
In this paper, we investigate the performance of a single-relay cooperative scenario where the source, relay and destination terminals are equipped with multiple transmit/receive antennas. We assume that conventional space-time block codes are employed in the underlying source-to-destination $(S \rightarrow D)$, source-to-relay $(S \rightarrow R)$ and relay-to-destination $(R \rightarrow D)$ links, and consider both decode-and-forward (DaF) and amplifyand-forward (AaF) relaying techniques. For the latter one, we consider two variants based on the availability of channel state information (CSI); namely, blind AaF and CSI-assisted AaF. Through the derivation of pairwise error probability, we quantify analytically the impact of multiple antenna deployment for each relaying technique under various scenarios which involve relay location and power control assumptions imposed on cooperating nodes. Our transmission model assumes that the source and destination terminals are equipped with $M_{S}$ transmit and $N$ receive antennas, respectively, and the relay terminal is equipped with $M_{R}$ receive and $M_{T}$ transmit antennas. For a scenario where $R \rightarrow D$ and $S \rightarrow D$ links are balanced and $S \rightarrow R$ link experiences sufficiently large $S N R$, our performance analysis demonstrates that the maximum achievable diversity order is $M_{T} \min \left(M_{S}, N\right)+M_{S} N$ for blind $\mathbf{A a F}$ scheme and $N\left(M_{T}+M_{S}\right)$ for both CSI-assisted AaF and DaF schemes. For another scenario where $R \rightarrow D$ link has a sufficiently large SNR and $S \rightarrow R$ and $S \rightarrow D$ links are balanced, CSI-assisted AaF, blind AaF and DaF schemes achieve diversity orders of $M_{S}\left(N+M_{R}\right), M_{S}\left(N+M_{T}\right)$, and $M_{s} N$, respectively. Other scenarios involving the availability of non-fading $R \rightarrow D$ link and poor inter-user channel quality are further investigated. An extensive Monte Carlo simulation study is also presented to corroborate the analytical results and to provide detailed performance comparisons among the three relaying techniques under consideration.
\end{abstract}

Index Terms-Cooperative diversity, space-time block coding, multiple antennas, fading channels, pairwise error probability.

\section{INTRODUCTION}

$\mathbf{T}$ RANSMISSION over wireless channels suffers from fading induced by multipath propagation which causes random fluctuations in the received signal level. A common approach to mitigate the degrading effects of fading is the use of diversity techniques. Most popular diversity forms are spatial diversity, temporal diversity, and frequency diversity. Among those, spatial diversity is particularly attractive because it is cheaper than the ones obtained in time and/or frequency and

Manuscript received November 17, 2006; revised June 16, 2007 and January 11, 2008; accepted January 22, 2008. The associate editor coordinating the review of this paper and approving it for publication was J. Andrews.

H. Muhaidat was with the Department of Electrical and Computer Engineering, University of Toronto, Canada. He is currently with the School of Engineering Science, Simon Fraser University, Burnaby, BC, Canada V5A 1S6 (e-mail: muhaidat@ieee.org).

M. Uysal is with the Dept. of Electrical Engr., University of Waterloo, Waterloo, Canada N2L 3G1 (e-mail: muysal@ece.uwaterloo.ca).

Digital Object Identifier 10.1109/TWC.2008.060952. can be combined with the latter diversity techniques. The conventional way of exploiting spatial diversity is to employ multiple co-located antennas at the transmitter and/or at the receiver. Through the deployment of suitably designed spacetime codes [1]-[3] diversity and/or multiplexing gains can be achieved at no cost in terms of transmission time and bandwidth expansion.

An alternative form of spatial diversity referred as "cooperative diversity" [4]-[8] has been recently proposed to realize diversity advantages in a distributed manner. Cooperative diversity exploits the broadcast nature of wireless transmission and creates a virtual (distributed) antenna array through cooperating nodes. In cooperative diversity (which is also known as user cooperation), the source terminal is assigned one or more partner terminals (relays) which are willing to share their physical resources. The relays process the signals overheard from the source terminal and retransmit them toward the destination. Two common relaying techniques are decodeand-forward (DaF) and amplify-and-forward (AaF). In $\mathrm{DaF}$ relaying, the relay terminal decodes its received signal and then re-encodes it (possibly using a different codebook) for transmission to the destination. On the other hand, in $\mathrm{AaF}$ relaying, the relay terminal retransmits a scaled version of the received signal without any attempt to decode it. AaF relaying can be furthered categorized based on the availability of channel state information (CSI) at the relay terminal. In CSI-assisted AaF scheme [6], the relay uses instantaneous CSI of the $S \rightarrow R$ link to scale its received noisy signal before forwarding. This ensures that the same output power is maintained for each realization. On the other hand, blind AaF scheme [9], [10] does not have access to CSI and employs fixed power constraint. This ensures that an average output power is maintained, but allows for the instantaneous output power to be much larger than the average. Although blind $\mathrm{AaF}$ relaying is not expected to perform as good as the CSI-assisted counterpart, the elimination of channel estimation at the relay terminal promises low complexity implementation and makes it attractive from a practical point of view.

Besides the relaying technique, another design choice in cooperative diversity schemes is the cooperation protocol. In their pioneering work [8], Laneman et al. consider a user cooperation scenario where the source signal is transmitted to a destination terminal through $L-1$ half-duplex relay terminals and demonstrate that the receiver achieves a diversity order of $L$. Their cooperation protocol is built upon a twophase transmission scheme. In the first phase (i.e., broadcasting phase), the source broadcasts to the destination and relay terminals. In the second phase (i.e., relaying phase), 
the relays transmit processed version of their received signals to the destination using either orthogonal sub-channels (repetition based cooperative diversity) or the same sub-channel (space-time coded cooperative diversity). Space-time coded cooperative diversity uses conventional orthogonal space-time block codes (STBCs) in a distributed fashion among the relay nodes. Nabar et al. [10] establish a unified framework for user cooperation protocols in single-relay wireless networks. They quantify achievable performance gains for distributed schemes in an analogy to conventional co-located multiantenna configurations. Specifically, they analyze three different protocols named Protocol I, Protocol II, and Protocol III which correspond to traditional MIMO (multi-input-multioutput), SIMO (single-input-multi-output) and MISO (multiinput-single-output) schemes, respectively. In Protocol I, the source terminal communicates with the relay and destination terminals during the first signaling interval. In the second signaling interval, both the relay and source terminals communicate with the destination terminal. It has been demonstrated in an independent work by Azarian et al. [11] that such a protocol is optimum in terms of diversity-multiplexing tradeoff. Protocol I is referred in [11] as "non-orthogonal amplify and forward (NAF) protocol". In Protocol II, the source terminal communicates with the relay and destination terminals in the first time slot. In the second time slot, only the relay terminal communicates with the destination. Protocol II is basically the same cooperation protocol proposed by Laneman et al. in [8]. Protocol III is essentially similar to Protocol I except that the destination terminal does not receive from the source during the first time slot. It can be noticed from the descriptions of protocols that the signal transmitted to both the relay and destination terminals is the same over the two time slots in Protocol II. Therefore, classical space-time code construction does not apply to Protocol II. On the other hand, Protocol I and Protocol III can transmit different signals to the relay and destination terminals. Hence, the conventional STBCs can be easily applied to these protocols in a distributed fashion. It should be recalled that the use of STBC has been also proposed by Laneman et al. in [8] for Protocol II. Their proposed use of STBC however implements coding across the relay nodes assuming a scenario with more than one relay and differs from the STBC setup in [10] which involves the source terminal in a single-relay scenario.

While most of the current literature on user cooperation is built upon the assumption that user nodes are equipped with a single antenna, there have been some recent results which exploit further the benefits of multiple antenna deployment. It is fairly easy to deploy nodes with multiple antennas in infrastructure-based fixed relay networks [12] which has recently spawned a surge of interest in MIMO relaying [13]-[17]. In [13], Wang et al. derive upper and lower bounds on the capacity of MIMO relay channels and demonstrate significant capacity gains. Their work, however, is based on some idealistic assumptions such as full-duplex relays and availability of channel state information at the transmitter side. Yiu et al. [14] consider distributed STBC with multiple antennas at the relay and destination terminals extending their own work in [15]. Under the assumption that there are $L$ active relays each of which is equipped with
$M_{T}$ antennas, a destination node with $N$ antennas and an underlying distributed STBC of size $K \times L_{1}$ ( $K$ : Number of time slots, $L_{1} \geq L$ ), it has been shown in [14] that a diversity order of $\min \left(L_{1} N, M_{T} L N\right)$ can be achieved for $\mathrm{DaF}$ relaying. The set-up in [14] assumes an error-free source-torelay link and only focuses on the relaying phase. In [16], we have investigated the impact of receive diversity on the error rate performance of AaF relaying. Specifically, we have shown that the diversity order over the relaying path is governed by the link which has smaller diversity order. Jing et al. [17] consider AaF relaying and investigate the application of linear dispersion space-time codes across multiple-antenna nodes. Their conclusion on the diversity order bottleneck is consistent with our results reported in [16].

In this paper, we consider a single-relay cooperative network and present a comprehensive performance analysis to demonstrate the effect of multiple antennas for different relaying techniques. We investigate various scenarios which involve relay location and power control assumptions imposed on cooperating nodes. Specifically, we consider Protocol II with blind $\mathrm{AaF}, \mathrm{CSI}$-assisted $\mathrm{AaF}, \mathrm{DaF}$ relaying and derive pairwise error probability (PEP) expressions quantifying analytically the impact of multiple antenna deployment at the source, relay and/or destination terminals on the diversity order for each of the relaying methods under consideration. We consider a user cooperation scenario where the source and destination terminals are equipped with $M_{T}$ and $N$ antennas, respectively. The relay terminal is equipped with $M_{R}$ receive and $M_{T}$ transmit antennas. We further assume $M_{S}=M_{R}=M_{T}=M^{1}$ and employ conventional STBC for source-to-relay $(S \rightarrow R)$ and source-to-destination $(S \rightarrow D)$ links $^{2}$.

For the considered cooperative transmission model with multiple-antenna nodes, our diversity gain analysis which has been further verified through Monte-Carlo simulations demonstrates that

- DaF and CSI-assisted AaF schemes achieve a diversity order of $N\left(M_{T}+M_{R}\right)$ under the assumption that $S \rightarrow D$ and $R \rightarrow D$ links are balanced (i.e., $E_{S D} / N_{0}=$ $\left.E_{R D} / N_{0}\right)$ and sufficiently large SNR for the $S \rightarrow R$ link (i.e., $E_{S R} / N_{0}>E_{S D} / N_{0}$ ), where $E_{S D}, E_{S R}$, and $E_{R D}$ represent the average energies available at the source and relay terminals taking into account for possibly different path loss and shadowing effects in $S \rightarrow D, S \rightarrow R$ and $R \rightarrow D$ links, respectively. This typically corresponds to a scenario where relay is located close to source. Under the same assumptions, the diversity order of blind $\mathrm{AaF}$ relaying achieves a diversity order of $M_{T} \min \left(M_{S}, N\right)+M_{S} N$. Our results

\footnotetext{
${ }^{1}$ In practical scenarios, the same antenna elements can be used for transmission and reception, therefore, it is reasonable to assume $M_{R}=M_{T}$. It makes practical sense to further assume $M_{R}=M_{T}=M_{S}$ since source and relay terminals are cooperating nodes designed for a given application, therefore typically share the same physical features. The purpose of assigning different variables for antenna numbers is solely to distinguish their effect on the diversity order.

${ }^{2}$ We should reemphasize that Protocol II is distributed SIMO implementation. The deployment of conventional STBC for underlying links in our model should not be confused with either distributed STBC implementation of [10] where STBC is applied to Protocols I and III to implement distributed MIMO and MISO configurations for single-relay networks or distributed STBC use of [8] in Protocol II across the relay nodes for multi-relay networks.
} 


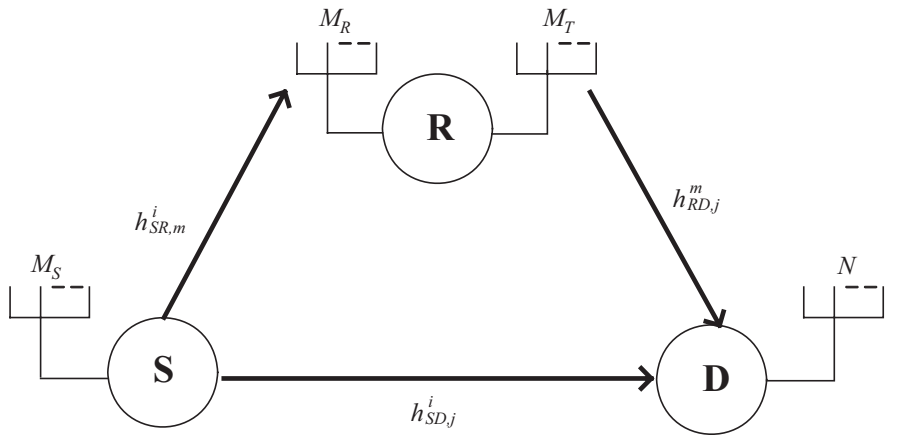

Fig. 1. Schematic representation of relay-assisted transmission.

indicate that $R \rightarrow D$ link determines the diversity order attributable to the relaying path (i.e., $N M_{T}$ ) for $\mathrm{DaF}$ and CSI-assisted $\mathrm{AaF}$ schemes while the minimum of diversity orders experienced in $S \rightarrow R$ and $R \rightarrow D$ links becomes the bottleneck for relaying path in the blind AaF scheme.

- CSI-assisted AaF, blind AaF, and DaF schemes achieve diversity orders of $M_{S}\left(N+M_{R}\right), M_{S}\left(N+M_{T}\right)$ and $N M_{S}$, respectively, under the assumption that $S \rightarrow D$ and $S \rightarrow R$ links are balanced and sufficiently large SNR for the $S \rightarrow R$ link (i.e., $E_{S R} / N_{0}>>E_{S D} / N_{0}$ ). This typically corresponds to a scenario where relay is located close to destination. Our results indicate that $S \rightarrow R$ link determines the diversity order attributable to the relaying path (i.e., $M_{S} M_{R}$ and $M_{S} M_{T}$ ) for CSI-assisted and blind $\mathrm{AaF}$ schemes. The diversity order of $\mathrm{DaF}$ relaying is limited to the non-cooperative scenario as it suffers from a weak $S \rightarrow R$ link due to relay's assumed location under the considered scenario.

The rest of the paper is organized as follows: In Section II, we describe our single-relay cooperative transmission model in conjunction with blind $\mathrm{AaF}$, CSI-assisted $\mathrm{AaF}$, and $\mathrm{DaF}$ relaying. In Section III, we present the PEP expressions and diversity order analysis for each of the relaying techniques under consideration. In Section IV, we provide an extensive Monte-Carlo simulation to corroborate the analytical results and to provide detailed performance comparisons among the competing schemes. Section V concludes the paper. The appendixes include mathematical details of the PEP derivations.

Notation: $(\cdot)^{*},(\cdot)^{\mathrm{T}}$, and $(\cdot)^{\mathrm{H}}$ denote conjugate, transpose, and Hermitian transpose operations, respectively. $\mathbf{I}_{Q}$ denotes the identity matrix of size $Q \times Q, \operatorname{tr}\{\cdot\}$ denotes a trace of a matrix, E[·] denotes expectation, $|\cdot|$ denotes the absolute value, $\|\cdot\|$ denotes the Euclidean norm of a vector. Bold uppercase letters denote matrices and bold lower-case letters denote vectors.

\section{TRANSMission MODEL}

We consider a wireless communication scenario where the source terminal $S$ transmits information to the destination terminal $D$ with the assistance of a single relay terminal $R$ (c.f. Fig.1). Whether or not the CSI of $S \rightarrow R$ link is available at the relay terminal depends on the relaying technique: In blind $\mathrm{AaF}$ relaying, no CSI is available at the relay while $\mathrm{DaF}$ and CSI-assisted $\mathrm{AaF}$ assume perfect CSI at the relay terminal. In
CSI-assisted AaF, the destination terminal is assumed to have perfect CSI of $S \rightarrow D, S \rightarrow R$, and $R \rightarrow D$ links. On the other hand, in blind AaF relaying, the destination terminal has CSI of $S \rightarrow D$ and concatenated $S \rightarrow R \rightarrow D$ links, but does not need explicit access to $S \rightarrow R$ link fading coefficient. In DaF relaying, the destination terminal has CSI of $S \rightarrow D$ and $S \rightarrow R \rightarrow D$ links. Our transmission model is built upon Protocol II [8], [10]: In the first $K$ time slots, i.e., broadcast phase, the source terminal communicates with the relay and destination terminals relying on an orthogonal STBC [2], [3] with a rate of $Q / K$ designed for $M$ transmit antennas ${ }^{3}$. In the next $\delta K$ time slots, i.e., relaying phase, only the relay terminal communicates with the destination relying on the same orthogonal STBC ${ }^{4}$. Here, $\delta=1$ for CSI-assisted AaF and $\mathrm{DaF}$, and $\delta=M_{T}$ for blind AaF relaying. The destination then performs maximum likelihood (ML) decoding on the signals received from the source and relay over $(\delta+1) K$ time slots.

Let $h_{S D, j}^{i}, h_{S R, m}^{i}$, and $h_{R D, j}^{m}$ respectively denote the complex fading coefficients over $S \rightarrow D$ link from the $i^{t h}$ transmit antenna to the $j^{t h}$ receive antenna, $S \rightarrow R$ link from the $i^{t h}$ transmit antenna to the $m^{\text {th }}$ receive antenna, and $R \rightarrow D$ link from the $m^{t h}$ transmit antenna to the $j^{t h}$ receive antenna. These channel coefficients are modeled as zero-mean complex Gaussian with variance 0.5 per dimension leading to the well-known Rayleigh fading channel model. Unless otherwise indicated, the variables $n$ (regardless of index) are independent and identically distributed (i.i.d.) zero mean complex Gaussian samples with $N_{0} / 2$ per dimension and model the additive noise. The received signals during the broadcasting phase at the $j^{\text {th }}(j=1,2, \ldots, N)$ receive antenna of the destination terminal are given by

$$
r_{D, j}^{k}=\sqrt{\frac{E_{S D}}{M_{S}}} \sum_{i=1}^{M_{S}} h_{S D, j}^{i} x_{i, k}+n_{D, j}^{k}, k=1,2, \ldots, K,
$$

where $x_{i, k}$ is the STBC-encoded modulation symbol sent from the $i^{t h}$ transmit antenna in time interval $k$. Similarly, the received signals at the $m^{\text {th }}\left(m=1,2, \ldots, M_{R}\right)$ receive antenna of the relay terminal are given by

$$
r_{R, m}^{k}=\sqrt{\frac{E_{S R}}{M_{S}}} \sum_{i=1}^{M_{S}} h_{S R, m}^{i} x_{i, k}+n_{R, m}^{k}, k=1,2, \ldots, K .
$$

In matrix notation, we can rewrite (2) as

$$
\mathbf{r}_{R, m}=\sqrt{\frac{E_{S R}}{M_{S}}} \mathbf{H}_{S R, m} \mathbf{x}+\mathbf{n}_{R, m},
$$

where $\mathbf{H}_{S R, m}$ is the $S \rightarrow R$ link channel matrix with size $K \times Q, \mathbf{x}=\left[\begin{array}{lll}x_{1} & \cdots & x_{Q}\end{array}\right]^{\mathrm{T}}$ denotes the codeword vector, and $\mathbf{n}_{R, m}=\left[\begin{array}{lll}n_{R, m}^{1} & \cdots & n_{R, m}^{K}\end{array}\right]^{\mathrm{T}}$ represents the noise vector. During the relaying phase, the received signals processed at the relay terminal are forwarded to the destination terminal. The type of processing depends on the deployed relaying technique. In the following, we present the signal models for CSI-assisted $\mathrm{AaF}$, blind $\mathrm{AaF}$, and $\mathrm{DaF}$ relaying techniques.

\footnotetext{
${ }^{3} Q$ symbols are sent over $K$ time slots using $M$ transmit antennas.

${ }^{4}$ As earlier noted, STBC is employed only in broadcasting phase for blind AaF relaying.
} 


\section{A. CSI-assisted AaF relaying}

In CSI-assisted AaF relaying, the relay terminal is assumed to have perfect knowledge of $S \rightarrow R$ fading coefficients. The relay terminal first performs spatio-temporal filtering and then combines the resulting signals to yield

$$
\mathbf{y}_{m}^{\prime}=\frac{1}{\beta_{S R}} \sum_{m=1}^{M_{R}} \mathbf{H}_{S R, m}^{\mathrm{H}} \mathbf{r}_{R, m}=\left(\beta_{S R, m} \times \mathbf{I}_{Q}\right) \mathbf{x}+\eta_{m},
$$

where $\beta_{S R}=v^{1 / 2}\left(\sum_{m=1}^{M_{R}} \sum_{i=1}^{M_{S}}\left|h_{S R, m}^{i}\right|^{2}\right)^{1 / 2}$ with $v$ as a constant which depends on the choice of the orthogonal code ${ }^{5}$. Alternatively, we can write (4) as

$$
y_{q}^{\prime}=\sqrt{\frac{E_{S R}}{M_{S}}} \beta_{S R} x_{q}+\eta_{q, m}, \quad q=1,2, \ldots Q,
$$

where $x_{q}$ denotes the $q^{\text {th }}$ entry of the data vector $\mathbf{x}$ and $\eta_{q}$ is the $q^{t h}$ entry of the filtered noise vector $\boldsymbol{\eta}=\left[\eta_{1}, \ldots, \eta_{Q}\right]^{6}$ which is still complex Gaussian with zero-mean and variance $N_{0} / 2$ per dimension. After scaling (5) by $\left(\beta_{S R}^{2} E_{S R} / M_{S}+\right.$ $\left.N_{0}\right)^{1 / 2}$ to ensure the unity of average energy at the relay output, we obtain

$$
y_{q}^{\prime \prime}=\sqrt{\frac{E_{S R} / M_{S}}{\beta_{S R}^{2} E_{S R} / M_{S}+N_{0}}} \beta_{S R} x_{q}+\frac{1}{\sqrt{\beta_{S R}^{2} E_{S R} / M_{S}+N_{0}}} \eta_{q} .
$$

The resulting signals $y_{1}^{\prime \prime}, \quad y_{2}^{\prime \prime}, \ldots, y_{Q}^{\prime \prime}$ are encoded by an STBC with rate of $Q / K$ and sent to the destination terminal. Let $y_{m, l}$ denote the STBC-encoded modulation symbols transmitted from the $m^{t h}$ antenna at time slot $l$. Noting $\delta=1$, the received signals at the destination terminal are given by

$$
r_{D, j}^{l}=\sqrt{\frac{E_{R D}}{M_{T}}} \sum_{m=1}^{M_{T}} h_{R D, j}^{m} y_{m, l}+n_{D, j}^{l}
$$

for $l=K+1, K+2, \ldots, 2 K$, and $j=1,2, \ldots, N$. The destination terminal applies spatio-temporal matched filtering to the received signal vector, i.e., multiplying $\mathbf{r}_{D, j}=$ $\left[\begin{array}{ccc}r_{D, j}^{K+1} & \ldots & r_{D, j}^{2 K}\end{array}\right]$ with $\left(1 / \beta_{R D, j}\right) \mathbf{H}_{R D, j}^{\mathrm{H}}$ where $\mathbf{H}_{R D, j}$ is the channel matrix of size $K \times Q$ and $\beta_{R D, j}=$ $\left(\nu\left(\left|h_{R D, j}^{1}\right|^{2}+\left|h_{R D, j}^{2}\right|^{2}, \ldots+\left|h_{R D, j}^{M_{T}}\right|^{2}\right)\right)^{1 / 2}$. This yields

$r_{D, j}^{q}=\sqrt{\frac{E_{R D}}{M_{T}}} \beta_{R D, j} y_{q}^{\prime \prime}+\tilde{\eta}_{j}^{q}, \quad q=1, \ldots, Q, j=1, \ldots, N$

where the filtered noise $\tilde{\eta}_{j}^{q}$ is still zero-mean complex Gaussian. Replacing (6) in (8), we obtain

$r_{D, j}^{q}=\sqrt{\frac{E_{S R} E_{R D}}{M_{T} M_{S}\left(\beta_{S R}^{2} E_{S R} / M_{S}+N_{0}\right)}} \beta_{R D, j} \beta_{S R} x_{q}+\widehat{n}_{D, j}^{q}$,

where the effective noise term is defined as

$$
\widehat{n}_{D, j}^{q}=\sqrt{\frac{E_{R D}}{M_{T}\left(\beta_{S R}^{2} E_{S R} / M_{S}+N_{0}\right)}} \beta_{R D, j} \eta_{q}+\tilde{\eta}_{j}^{q}
$$

\footnotetext{
${ }^{5}$ For Alamouti scheme, $v=1$. For G3 and G4 codes of [2], $v=2$

${ }^{6}$ The filtered noise vector is given by $\boldsymbol{\eta}=\left[\eta_{1}, \ldots, \eta_{q}\right]^{\mathrm{T}}=$ $\left(1 / \beta_{S R}\right) \sum_{m=1}^{M_{R}} \mathbf{H}_{S R, m}^{\mathrm{H}} \mathbf{n}_{R, m}$. Due to the orthogonality of the channel matrix $\mathbf{H}_{S R, m}$, the entries of $\boldsymbol{\eta}$ are still white, i.e., $E\left[\boldsymbol{\eta} \boldsymbol{\eta}^{\mathrm{H}}\right]=N_{0} \mathbf{I}_{Q}$.
}

which is complex Gaussian (conditioned on $\beta_{S R, m}$ and $\beta_{R D, j}$ ) with zero mean and variance of

$$
\begin{aligned}
E\left[\left|\widehat{n}_{D, j}^{q}\right|^{2}\right. & \left.\mid \beta_{R D, j}, \beta_{S R, m}\right]=N_{0} \\
& \times\left(1+\frac{E_{R D} \beta_{R D, j}^{2}}{M_{T}\left(\beta_{S R}^{2} E_{S R} / M_{S}+N_{0}\right)}\right) .
\end{aligned}
$$

The destination terminal normalizes the received signal given by (8) with

$$
\left(1+\frac{\beta_{R D, j}^{2} E_{R D}}{M_{T}\left(\beta_{S R}^{2} E_{S R} / M_{S}+N_{0}\right)}\right)^{1 / 2},
$$

resulting in

$\tilde{r}_{D, j}^{q}=\sqrt{\alpha_{j}} \beta_{R D, j} \beta_{S R} x_{q}+\tilde{n}_{D, j}^{q}, q=1,2, \ldots Q, j=1,2, \ldots N$

where $\alpha_{j}$ is defined by

$$
\alpha_{j}=\frac{\left(E_{S R} / N_{0}\right) E_{R D}}{\left(M_{T} \beta_{S R}^{2}\left(E_{S R} / N_{0}\right)+M_{S} M_{T}\right)+M_{S} \beta_{R D, j}^{2}\left(E_{R D} / N_{0}\right)} .
$$

The received signals at $N$ receive antennas of the destination terminal, i.e., $\tilde{r}_{D, j}^{q}, j=1, \ldots, N$ are then fed to the ML decoder.

\section{B. Blind AaF relaying}

In blind AaF relaying, the relay terminal does not have the knowledge of the $S \rightarrow R$ fading coefficients. Therefore, unlike CSI-assisted AaF relaying, coherent combining of received space-time coded signals through spatio-temporal filtering is not feasible. Here, we simply assume that the relay forwards the received signals after proper normalization. Each of the received signal, i.e. $r_{R, m}^{k}$, is first normalized by a factor of $\sqrt{E\left[\left|r_{R, m}^{k}\right|\right]}=\sqrt{E_{S R} / M_{S}+N_{0}}$ to ensure the unity of average energy and then transmitted through $M_{T}$ antennas consecutively during the next $\delta K$ time slots. The received signal at the destination terminal from the $t^{t h}\left(t=1,2, \ldots, M_{T}\right)$ antenna is given by

$$
r_{D, j}^{t, l}=\sqrt{\frac{E_{R D}}{M_{T}}} h_{R D, j}^{t} \frac{r_{R, m}^{k}}{\sqrt{E_{S R} / M_{S}+N_{0}}}+n_{D, j}^{t, l},
$$

for $l=t K+1, \ldots, t(K+1), k=l-t K$ and $j=1, \ldots, N$. Replacing (2) in (14) and noting $m=t$, we obtain

$$
\begin{gathered}
r_{D, j}^{t, l}=\sqrt{\frac{E_{S R} E_{R D}}{M_{T} M_{S}\left(E_{S R} / M_{S}+N_{0}\right)}} h_{R D, j}^{t} \\
\sum_{i=1}^{M_{S}} h_{S R, t}^{i} x_{i, k}+\widehat{n}_{D, j}^{t, l},
\end{gathered}
$$

where the effective noise term $\widehat{n}_{D, j}^{t, l}$ is given as

$$
\widehat{n}_{D, j}^{t, l}=\sqrt{\frac{E_{R D}}{M_{T}\left(E_{S R} / M_{S}+N_{0}\right)}} h_{R D, j}^{t} n_{R, t}^{k}+n_{D, j}^{t, l}
$$

which is complex Gaussian (conditioned on $h_{R D, j}^{t}$ ) with zero mean and variance of 


$$
\begin{aligned}
& E\left[\left|\widehat{n}_{D, j}^{t, l}\right|^{2} \mid h_{R D, j}^{t}\right]=N_{0} \\
& \times\left(1+\frac{E_{R D}}{M_{T}\left(E_{S R} / M_{S}+N_{0}\right)}\left|h_{R D, j}^{t}\right|^{2}\right) .
\end{aligned}
$$

The destination terminal normalizes the received signal given by (15) with

$$
\left(1+\frac{E_{R D}}{M_{T}\left(E_{S R} / M_{S}+N_{0}\right)}\left|h_{R D, j}^{t}\right|^{2}\right)^{1 / 2},
$$

resulting in

$$
r_{D, j}^{t, l}=\sqrt{\gamma_{j}^{t}} \sqrt{E_{R D}} h_{R D, j}^{t} \sum_{i=1}^{M_{S}} h_{S R, t}^{i} x_{i, k}+\tilde{n}_{D, j}^{t, l},
$$

where $\tilde{n}_{D, j}^{t, l}$ turns out to be zero-mean complex Gaussian with variance $N_{0} / 2$ per dimension and the scaling factor $\gamma_{j}^{t}$ is defined as

$$
\gamma_{j}^{t}=\frac{E_{S R}}{N_{0}} \frac{1}{\left(M_{T} M_{S}+M_{T} \frac{E_{S R}}{N_{0}}+M_{S}\left|h_{R D, j}^{t}\right|^{2} \frac{E_{R D}}{N_{0}}\right)} .
$$

The received signals at $N$ receive antennas of the destination terminal, i.e., $r_{D, j}^{t, l}, j=1, \ldots, N$ are then fed to the $\mathrm{ML}$ decoder.

\section{DaF relaying}

Conventional DaF relaying is known to suffer from error propagation leading to error floors if SNR in the $S \rightarrow R$ link is poor. There have been several attempts in the literature to improve the performance of DaF protocol. Among others, Laneman et al. [6] have considered adaptive DaF relaying in which the relay terminal decodes the received signals if the instantaneous SNR at the relay terminal is greater than a certain threshold. Such an adaptive relaying technique overcomes the main limitations of fixed conventional relaying although no optimality is claimed. Recently, in [11], Azarian et al. have proposed a dynamic $\mathrm{DaF}$ protocol where the relay decodes only if the accumulated mutual information between its received signal and the source signal exceeds a certain rate. They have shown that the proposed protocol is optimum in a certain range of multiplexing gains. In our work, mainly due to mathematical convenience, we consider the adaptive $\mathrm{DaF}$ relaying proposed in [6]. We assume that instantaneous SNR values of the underlying links are available at the destination terminal. The threshold value is chosen as $\gamma_{t h}=$ $\gamma_{S D}+\gamma_{R D}$ where $\gamma_{S D}=\sum_{j=1}^{N} \sum_{i=1}^{M_{S}}\left|h_{S D, j}^{i}\right|^{2} E_{S D} / N_{0}$ and $\gamma_{R D}=\sum_{j=1}^{N} \sum_{m=1}^{M_{T}}\left|h_{R D, j}^{m}\right|^{2} E_{R D} / N_{0}$ are the received instantaneous SNRs of $S \rightarrow D$ and $R \rightarrow D$ links, respectively. If this condition is satisfied, the destination terminal asks the relay to engage in forwarding. The relay feeds its decoded data into an STBC-encoder for transmission to the destination terminal. In this case, the received signals at the destination terminal can be written as

$$
r_{D, j}^{l}=\sqrt{\frac{E_{R D}}{M_{T}}} \sum_{m=1}^{M_{T}} h_{R D, j}^{m} y_{m, l}+n_{D}^{l},
$$

for $l=K+1, \ldots, 2 K$, and $j=1, \ldots, N$. In (20), $y_{m, l}$ denotes the STBC-encoded modulation symbol transmitted from the relay's $m^{t h}$ transmit antenna in time slot $l$. The received signals at $N$ receive antennas of the destination terminal, i.e., $r_{D, j}^{l}, j=1, \ldots, N$ are then fed to the ML decoder.

\section{Diversity GAin AnAlysis}

In this section, we investigate the achievable diversity gains (orders) for each of the considered relaying techniques through the derivation of PEP expressions. Diversity order is defined as the negative of the asymptotic slope of the PEP [1]. Defining the transmitted codeword vector from the source and the erroneously-decoded codeword vector at the destination terminal, respectively, as $\mathbf{x}=\left[x_{1}, \ldots, x_{Q}\right]^{T}$ and $\hat{\mathbf{x}}=\left[\hat{x}_{1}, \ldots, \hat{x}_{Q}\right]^{T}$, the conditional PEP is given by

$$
\begin{gathered}
P\left(\mathbf{x}, \hat{\mathbf{x}} \mid h_{S R, m}^{i}, h_{R D, j}^{m}, h_{S D, j}^{i}, i=1, \ldots, M_{S}, m=1, \ldots, M_{T},\right. \\
j=1, \ldots, N)=Q\left(\sqrt{\frac{d^{2}(\mathbf{x}, \hat{\mathbf{x}})}{2 N_{0}}}\right)
\end{gathered}
$$

assuming ML decoding. Here, $Q($.$) is the Gaussian- Q$ function and $d^{2}(\mathbf{x}, \hat{\mathbf{x}})$ denotes the Euclidean distance between $\mathbf{x}$ and $\hat{\mathbf{x}}$. Applying the standard Chernoff bound to (21), we obtain

$$
\begin{gathered}
P\left(\mathbf{x}, \hat{\mathbf{x}} \mid h_{S R, m}^{i}, h_{R D, j}^{m}, h_{S D, j}^{i}, i=1, \ldots, M_{S}, m=1, \ldots, M_{T},\right. \\
j=1, \ldots, N) \leq \exp \left(-\frac{d^{2}(\mathbf{x}, \hat{\mathbf{x}})}{4 N_{0}}\right) .
\end{gathered}
$$

\section{A. PEP for CSI-assisted AaF relaying}

The Euclidean distance $d^{2}(\mathbf{x}, \hat{\mathbf{x}})$ for AaF relaying can be written as

$$
\begin{aligned}
& d^{2}(\mathbf{x}, \hat{\mathbf{x}})=d_{S \rightarrow R \rightarrow D}^{2}(\mathbf{x}, \hat{\mathbf{x}})+d_{S \rightarrow D}^{2}(\mathbf{x}, \hat{\mathbf{x}}) \\
& =\frac{\lambda E_{S D}}{M_{S}} \sum_{j=1}^{N} \sum_{i=1}^{M_{S}}\left|h_{S D, j}^{i}\right|^{2}+\lambda \sum_{j=1}^{N} \alpha_{j} \sum_{m=1}^{M_{T}}\left|h_{R D, j}^{m}\right|^{2} \beta_{S R}^{2}
\end{aligned}
$$

where $\alpha_{j}$ is earlier defined by (13), $\lambda=$ $v\left|x_{1}-\hat{x}_{1}\right|^{2}+\ldots+v\left|x_{Q}-\hat{x}_{Q}\right|^{2}$ denotes the eigenvalue of the codeword difference matrix, and $\beta_{S R}=v^{1 / 2}\left(\sum_{m=1}^{M_{R}} \sum_{i=1}^{M_{S}}\left|h_{S R, m}^{i}\right|^{2}\right)^{1 / 2}$. Since $\alpha_{j}$ is a function of $\beta_{R D, j}^{2}$ term, the derivation of a general PEP expression becomes analytically difficult without any assumptions imposed on the SNR in the underlying links. In the following, we consider various power control scenarios and analyze the resulting diversity orders.

Scenario 1 (Balanced $S \rightarrow D$ and $R \rightarrow D$ links and high SNR in $S \rightarrow R$ link ): We assume that $S \rightarrow R$ link experiences a high SNR which corresponds to a practical scenario where relay is located close the source. Conditioned on $\left|h_{R D, j}^{m}\right|$, the noise terms at different receive antennas of the destination terminal, c.f., (10), are still Gaussian, but no longer spatially white. However, due to the high SNR assumption in the link, we can safely assume that the correlation of noise terms is negligible. We further assume power control between source and relay terminals such that $S \rightarrow D$ and $R \rightarrow D$ links are balanced, i.e., $E_{S R} / N_{0}>>E_{S D} / N_{0}=E_{R D} / N_{0}$. 
Under these assumptions, the scaling factor in (13) reduces to $\alpha_{j}=E_{R D}\left(M_{T} \beta_{S R}^{2}\right)^{-1}$. Hence, (23) is simplified as

$d^{2}(\mathbf{x}, \hat{\mathbf{x}})=\frac{\lambda E_{S D}}{M_{S}} \sum_{j=1}^{N} \sum_{i=1}^{M_{S}}\left|h_{S D, j}^{i}\right|^{2}+\frac{\lambda E_{S D}}{M_{T}} \sum_{j=1}^{N} \sum_{m=1}^{M_{T}}\left|h_{R D, j}^{m}\right|^{2}$.

Substituting (24) in (22) and taking the expectation with respect to $\left|h_{R D, j}^{m}\right|$ and $\left|h_{S D, j}^{i}\right|$ which are Rayleigh distributed and assuming $E_{S D} / N_{0}>>1$, we find PEP as

$$
P(\mathbf{x}, \hat{\mathbf{x}}) \leq\left(\frac{E_{S D}}{4 M N_{0}}\right)^{-N\left(M_{S}+M_{T}\right)} \lambda^{-N\left(M_{S}+M_{T}\right)} .
$$

It is observed from (25) that the diversity order achieved by CSI-assisted AaF relaying in this scenario is $N\left(M_{S}+M_{T}\right)=2 N M$.

Scenario 2 (Balanced $S \rightarrow D$ and $S \rightarrow R$ links and high SNR in $R \rightarrow D$ link): We now assume that $R \rightarrow D$ link experiences a high SNR which is likely to occur in practical scenarios when the relay is close to the destination terminal. We further assume that $S \rightarrow D$ and $S \rightarrow R$ links are balanced, i.e., $E_{R D} / N_{0}>>E_{S D} / N_{0}=E_{S R} / N_{0}$. As in Scenario 1 , the noise terms at different receive antennas of the destination terminal are spatially correlated. Thus, to simplify performance analysis, we assume that the number of receive antennas at the destination terminal seen by the $S \rightarrow R \rightarrow D$ link is equal to unity ${ }^{7}$. Under this assumption, the scaling factor in (13) can be approximated as $\alpha \approx E_{S R} / M_{S} \beta_{R D}^{2}$, therefore (23) reduces to

$$
d^{2}(\mathbf{x}, \hat{\mathbf{x}})=\frac{\lambda E_{S D}}{M_{S}} \sum_{j=1}^{N} \sum_{i=1}^{M_{S}}\left|h_{S D, j}^{i}\right|^{2}+\frac{\lambda E_{S R}}{M_{S}} \sum_{m=1}^{M_{R}} \sum_{i=1}^{M_{S}}\left|h_{S R, m}^{i}\right|^{2} .
$$

Substituting (26) in (22), taking the expectation with respect to $\left|h_{S D, j}^{i}\right|$ and $\left|h_{S R, m}^{i}\right|$ which are Rayleigh distributed, and assuming $E_{S D} / N_{0}>>1$, we find PEP as

$$
P(\mathbf{x}, \hat{\mathbf{x}}) \leq\left(\frac{E_{S D}}{4 M_{S} N_{0}}\right)^{-\left(N M_{S}+M_{R} M_{S}\right)} \lambda^{-\left(N M_{S}+M_{R} M_{S}\right)} .
$$

The achievable diversity order in this scenario is therefore $M_{S}\left(N+M_{R}\right)=N M+M^{2}$. This indicates that the diversity order achieved in Scenario 2 is higher than that of Scenario 1 provided that $M>N$.

Scenario 3 (Poor SNR in $S \rightarrow R$ link): In Scenario 1 , we have assumed that SNR in $S \rightarrow R$ link is sufficiently large, i.e., $E_{S R} / N_{0}>>E_{S D} / N_{0}=E_{R D} / N_{0}$. Now, we consider the limiting case of $E_{S R} / N_{0} \rightarrow 0$ (we still assume $\left.E_{S D} / N_{0}=E_{R D} / N_{0}>>1\right)$. Under this scenario, $d^{2}(\mathbf{x}, \hat{\mathbf{x}})$ is given by (26). Following similar steps as in Scenario 2, we obtain the PEP as

$$
P(\mathbf{x}, \hat{\mathbf{x}}) \leq\left(\frac{E_{S D}}{4 M_{S} N_{0}} \lambda\right)^{-N M_{S}}\left(1+\frac{E_{S R}}{4 M_{S} N_{0}} \lambda\right)^{-M_{R} M_{S}} .
$$

\footnotetext{
${ }^{7}$ It has been observed through a Monte-Carlo simulation experiment that this assumption does not affect the diversity order since the number of antennas seen by $S \rightarrow D$ link becomes the determining factor for this scenario.
}

Due to the limiting case of $E_{S R} / N_{0} \rightarrow 0$, the first term in this case evidently dominates the performance. Therefore, the diversity order is limited to the diversity of the noncooperative case given by $N M_{S}$.

Scenario 4 (Non-fading $R \rightarrow D$ link): Now, we focus on the case where the channel between the relay and the destination terminals is AWGN, i.e., $h_{R D}=1$. Physically, this assumption corresponds to a case where the destination and relay terminals are static and have a very strong line-of-sight connection. Further assuming that $S \rightarrow D$ and $R \rightarrow D$ links are balanced and high SNR in the $S \rightarrow R$ link, $d^{2}(\mathbf{x}, \hat{\mathbf{x}})$ reduces to

$$
d^{2}(\mathbf{x}, \hat{\mathbf{x}})=\frac{\lambda E_{S D}}{M_{S}} \sum_{j=1}^{N} \sum_{i=1}^{M_{S}}\left|h_{S D, j}^{i}\right|^{2}+\lambda N E_{S D} .
$$

Substituting (29) in (22) and taking the expectation with respect to $\left|h_{S D, j}^{i}\right|$ which is Rayleigh distributed, we find PEP as

$$
P(\mathbf{x}, \hat{\mathbf{x}}) \leq\left(\frac{E_{S D}}{4 M_{S} N_{0}}\right)^{-N M_{S}} \lambda^{-N M_{S}} \exp \left(-N \lambda \frac{E_{S D}}{4 N_{0}}\right) .
$$

Here, the exponential term becomes dominant and, therefore, the diversity order is large and can not be determined by an integer value anymore, i.e., an AWGN-like performance is observed.

\section{B. PEP for blind AaF relaying}

Given (1) and (18), the Euclidean distance $d^{2}(\mathbf{x}, \hat{\mathbf{x}})$ for blind $\mathrm{AaF}$ relaying can be written as

$$
\begin{array}{r}
d^{2}(\mathbf{x}, \hat{\mathbf{x}})=\lambda E_{R D} \sum_{j=1}^{N} \sum_{t=1}^{M_{T}} \gamma_{j}^{t}\left|h_{R D, j}^{t}\right|^{2} \sum_{i=1}^{M_{S}}\left|h_{S R, t}^{i}\right|^{2} \\
+\frac{\lambda E_{S D}}{M_{S}} \sum_{j=1}^{N} \sum_{i=1}^{M_{S}}\left|h_{S D, j}^{i}\right|^{2} .
\end{array}
$$

Similar to the CSI-assisted AaF relaying case, the derivation of a general PEP expression becomes difficult without any assumptions imposed on the SNRs of the underlying links. In the following, we consider the aforementioned scenarios in the previous section and analyze the resulting diversity orders.

Scenario 1 (High SNR in $S \rightarrow R$ link and balanced $S \rightarrow D$ and $R \rightarrow D$ links): Following steps detailed in the Appendix, we obtain the PEP expressions as

$$
\begin{array}{lc}
P(\mathbf{x}, \hat{\mathbf{x}}) \leq\left(\frac{\Gamma\left(N-M_{S}\right)}{\Gamma(N)}\right)^{M_{T}}\left(\frac{\lambda E_{S D}}{4 M N_{0}}\right)^{-M_{S}\left(N+M_{T}\right)} & N>M_{S} \\
P(\mathbf{x}, \hat{\mathbf{x}}) \leq\left(\frac{\Gamma\left(M_{S}-N\right)}{\Gamma\left(M_{S}\right)}\right)^{M_{T}}\left(\frac{\lambda E_{S D}}{4 M N_{0}}\right)^{-N\left(M_{S}+M_{T}\right)} & M_{S}>N \\
P(\mathbf{x}, \hat{\mathbf{x}}) \leq \frac{\log ^{M_{T}\left(E_{S D} / N_{0}\right)}}{\Gamma(N)^{M} T}\left(\frac{\lambda E_{S D}}{4 M N_{0}}\right)^{-M_{S}\left(N+M_{T}\right)} & M_{S}=N .
\end{array}
$$

It can be observed from (32)-(34) that the maximum achievable diversity order is given by 


$$
P(\mathbf{x}, \hat{\mathbf{x}})_{\text {end-to-end }}=\left\{\begin{array}{l}
P_{S \rightarrow R}(\mathbf{x}, f(\mathbf{x})) P(f(\mathbf{x}), \hat{\mathbf{x}})+\left(1-P_{S \rightarrow R}(\mathbf{x}, f(\mathbf{x}))\right) P(\mathbf{x}, \hat{\mathbf{x}}) \text { for } \gamma_{S R} \geq \gamma_{t h} \\
P_{S \rightarrow D}(\mathbf{x}, \hat{\mathbf{x}}) \text { for } \gamma_{S R}<\gamma_{t h}
\end{array}\right.
$$

$$
P(\mathbf{x}, \hat{\mathbf{x}})_{\text {end-to-end }} \leq P_{S \rightarrow R}(\mathbf{x}, f(\mathbf{x}))+\left(1-P_{S \rightarrow R}(\mathbf{x}, f(\mathbf{x}))\right) P(\mathbf{x}, \hat{\mathbf{x}}) \quad \text { for } \gamma_{S R} \geq \gamma_{t h}
$$

$M_{T} \min \left(M_{S}, N\right)+M_{S} N$. This illustrates that the smaller of diversity orders experienced in $S \rightarrow R$ and $R \rightarrow D$ links becomes the performance bottleneck for the relaying path. Comparison to (25) further reveals that CSI-assisted $\mathrm{AaF}$ and blind AaF relaying yield the same diversity order, provided that $M_{S} \geqslant N$.

Scenario 2 (High SNR in $R \rightarrow D$ link and balanced $S \rightarrow D$ and $S \rightarrow R$ links): Under these assumptions, $\gamma_{j}^{t}$ can be approximated as $\gamma_{j}^{t} \approx E_{S R} / N_{0} /\left(M_{S}\left|h_{R D, j}^{t}\right|^{2} E_{R D} / N_{0}\right)$. Similar to our earlier analysis for CSI-assisted AaF, we assume the number of receive antennas seen by the $S \rightarrow R \rightarrow D$ link is equal to one to simplify the performance analysis. The Euclidean distance can be then written as

$d^{2}(\mathbf{x}, \hat{\mathbf{x}})=\frac{\lambda E_{S R}}{M_{S}} \sum_{t=1}^{M_{T}} \sum_{i=1}^{M_{S}}\left|h_{S R, t}^{i}\right|^{2}+\frac{\lambda E_{S D}}{M_{S}} \sum_{j=1}^{N} \sum_{i=1}^{M_{S}}\left|h_{S D, j}^{i}\right|^{2}$.

Defining $Y_{1}=\sum_{j=1}^{N} \sum_{i=1}^{M_{S}}\left|h_{S D, j}^{i}\right|^{2}, \quad Y_{2}=$ $\sum_{t=1}^{M_{T}} \sum_{i=1}^{M_{S}}\left|h_{S R, t}^{i}\right|^{2}$, and noting that $\left|h_{S D, j}^{i}\right|$ and $\left|h_{S R, t}^{i}\right|$ are Rayleigh distributed, their characteristic functions are readily available, c.f. (51). Replacing those in (50) and assuming $E_{S D} / N_{0}>>1$, we find the PEP as

$$
P(\mathbf{x}, \hat{\mathbf{x}}) \leq\left(\frac{E_{S D}}{4 M N_{0}} \lambda\right)^{-M_{S}\left(N+M_{T}\right)} .
$$

The diversity order in this scenario is $M_{S}\left(M_{T}+N\right)$ which is obviously either equal or larger than the diversity order observed for the previous scenario, i.e., $M_{T} \min \left(M_{S}, N\right)+M_{S} N$.

Scenario 3 (Poor SNR in $S \rightarrow R$ link): For the limiting case of $E_{S R} / N_{0} \rightarrow 0, d^{2}(\mathbf{x}, \hat{\mathbf{x}})$ is given by (35). Following similar steps as in Scenario 2, we find the PEP expression as

$$
P(\mathbf{x}, \hat{\mathbf{x}}) \leq\left(\frac{E_{S D}}{4 M N_{0}} \lambda\right)^{-N M_{S}}\left(1+\frac{\lambda E_{S R}}{4 M N_{0}}\right)^{-M_{T} M_{S}}
$$

Since $E_{S R} / N_{0} \rightarrow 0, d^{2}(\mathbf{x}, \hat{\mathbf{x}})$ is assumed, the first term becomes dominant. Therefore, it can be easily concluded that the diversity order in (37) is limited to $N M_{S}$ as observed for CSI-assisted case. This indicates that for poor inter-user channel, the performance of blind AaF relaying is limited to that of the non-cooperative case, i.e., direct transmission.

Scenario 4 (Non-fading $R \rightarrow D$ link): Under the assumptions of $E_{S D} / N_{0}=E_{R D} / N_{0}$ and $E_{S R} / N_{0}>E_{S D} / N_{0}$, $\gamma_{j}^{t}$ in (19) can be approximated as $\gamma_{j}^{t} \approx 1 / M_{T}$. In this case, $d^{2}(\mathbf{x}, \hat{\mathbf{x}})$ can be written as $d^{2}(\mathbf{x}, \hat{\mathbf{x}})=\frac{E_{S D}}{M_{S}} \sum_{j=1}^{N} \sum_{i=1}^{M_{S}}\left|h_{S D, j}^{i}\right|^{2} \lambda+N \frac{E_{S D}}{M_{T}} \sum_{t=1}^{M_{T}} \sum_{i=1}^{M_{S}}\left|h_{S R, t}^{i}\right|^{2} \lambda$.

Substituting (38) in (22), taking the expectation with respect to $\left|h_{S D, j}^{i}\right|$ and $\left|h_{S R, t}^{i}\right|$ which are Rayleigh distributed, and assuming $E_{S D} / N_{0}>>1$, we find PEP as

$$
P(\mathbf{x}, \hat{\mathbf{x}}) \leq \frac{M^{M_{S}\left(M_{T}+N\right)}}{N^{M_{T} M_{S}}}\left(\frac{\lambda E_{S D}}{4 N_{0}}\right)^{-M_{S}\left(M_{T}+N\right)} .
$$

It can be observed that the diversity order for a non-fading $R \rightarrow D$ link is $M_{S}\left(M_{T}+N\right)$. Comparison to (30) reveals that CSI-assisted AaF significantly performs better than blind AaF relaying under non-fading link assumption.

\section{PEP for DaF relaying}

For adaptive $\mathrm{DaF}$ relaying under consideration, end-to-end PEP is given by (40) at the top of this page. In (40), $f(\mathbf{x})$ denotes the relay output, $P(f(\mathbf{x}), \mathbf{x})$ is the PEP when cooperation is employed given that the codeword is decoded incorrectly at the relay terminal, $P(\mathbf{x}, \hat{\mathbf{x}})$ denotes the PEP when cooperation is employed given that the codeword is decoded correctly at the relay terminal, and $P_{S \rightarrow R}(\mathbf{x}, f(\mathbf{x}))$ is the PEP over the $S \rightarrow R$ link. For $\gamma_{S R}<\gamma_{t h}$, end-to-end PEP is simply restricted to $P_{S \rightarrow D}(\mathbf{x}, \hat{\mathbf{x}})$ which represents the PEP of the direct transmission. To simplify the performance analysis, we can upper bound $P(\mathbf{x}, \hat{\mathbf{x}})_{\text {end-to-end }}$ as given in (41) which can be found at the top of this page, where we have used fact $P(f(\mathbf{x}), \hat{\mathbf{x}}) \leq 1$.

Scenario 1 (High SNR in the $S \rightarrow R$ link and balanced $S \rightarrow D$ and $R \rightarrow D$ links): Since $E_{S R} / N_{0}>>E_{S D} / N_{0}$, we can assume that $P_{S \rightarrow R}(\mathbf{x}, f(\mathbf{x})) \rightarrow 0$. Therefore, (41) can be approximated as $P(\mathbf{x}, \hat{\mathbf{x}})_{\text {end-to-end }} \leq P(\mathbf{x}, \hat{\mathbf{x}})$. The Euclidean distance in this case is given as

$$
d^{2}(\mathbf{x}, \hat{\mathbf{x}})=\frac{E_{S D}}{M_{S}} \sum_{j=1}^{N} \sum_{i=1}^{M_{S}}\left|h_{S D, j}^{i}\right|^{2} \lambda+\frac{E_{S D}}{M_{T}} \sum_{j=1}^{N} \sum_{t=1}^{M_{T}}\left|h_{R D, j}^{t}\right|^{2} \lambda .
$$

Substituting (42) in (22), taking the expectation with respect to $\left|h_{S D, j}^{i}\right|$ and $\left|h_{R D, j}^{t}\right|$ which are Rayleigh distributed, and assuming $E_{S D} / N_{0}>>1$, we find PEP as

$P(\mathbf{x}, \hat{\mathbf{x}})_{e n d-t o-e n d} \leq\left(\frac{E_{S D}}{4 M N_{0}}\right)^{-N\left(M_{S}+M_{T}\right)} \lambda^{-N\left(M_{S}+M_{T}\right)}$ 
TABLE I

DIVERSITY ORDERS OF BLIND A $a \mathrm{~F}, \mathrm{CSI}$-ASSISTED A $a \mathrm{~F}$, AND D $a \mathrm{~F}$ RELAYING.

\begin{tabular}{|l|l|l|l|}
\hline Scenario & Blind $\mathrm{AaF}$ & CSI-assisted $\mathrm{AaF}$ & $\mathrm{DaF}$ \\
\hline 1 & $M_{T} \min \left(M_{S}, N\right)+M_{S} N$ & $\left(M_{S}+M_{T}\right)$ & $N\left(M_{S}+M_{T}\right)$ \\
\hline 2 & $M_{S}\left(N+M_{T}\right)$ & $M_{S}\left(N+M_{R}\right)$ & $N M_{S}$ \\
\hline 3 & $N M_{S}$ & $N M_{S}$ & $N M_{S}$ \\
\hline 4 & $M_{S}\left(N+M_{T}\right)$ & Large & Large \\
\hline
\end{tabular}

We observe that DaF relaying is able to achieve a diversity order of $N\left(M_{S}+M_{T}\right)$ which is the same as that of CSIassisted AaF relaying under the same scenario.

Scenario 2 (High SNR in the $R \rightarrow D$ link and balanced $S \rightarrow R$ and $S \rightarrow D$ links): This scenario imposes the assumption of $E_{S R} / N_{0}=E_{S D} / N_{0}<<E_{R D} / N_{0}$, therefore we have $P\left(\gamma_{S R}<\gamma_{t h}\right) \approx 1$. It can be therefore easily concluded that the diversity order is simply limited to the non-cooperative case, i.e., $M N$.

Scenario 3 (Poor SNR in the $S \rightarrow R$ link): When the inter-user channel has a very poor quality, we have $P\left(\gamma_{S R}<\gamma_{t h}\right) \approx 1$ and, therefore, $P(\mathbf{x}, \hat{\mathbf{x}})_{\text {end-to-end }}=P_{S \rightarrow D}(\mathbf{x}, \hat{\mathbf{x}})$. The final PEP is then obtained as

$$
P(\mathbf{x}, \hat{\mathbf{x}})_{\text {end-to-end }} \leq\left(\frac{E_{S D}}{4 M N_{0}}\right)^{-N M_{S}} \lambda^{-N M_{S}}
$$

Consequently, for poor inter-user channel, similar to the other two relaying protocols, the performance is again limited to the non-cooperative scenario.

Scenario 4 (Non-fading $R \rightarrow D$ link): Under the assumption of $E_{S R} / N_{0}>>E_{S D} / N_{0}$ and $E_{S D} / N_{0}=E_{R D} / N_{0}>>1$, we observe that $P\left(\gamma_{S R} \geq \gamma_{t h}\right) \approx 1$, hence, we can assume that $P_{S \rightarrow R}(\mathbf{x}, f(\mathbf{x})) \approx 0$ which yields $P(\mathbf{x}, \hat{\mathbf{x}})_{\text {end-to-end }} \leq P(\mathbf{x}, \hat{\mathbf{x}})$. For $h_{R D}=1$, the Euclidean distance is given as

$$
d^{2}(\mathbf{x}, \hat{\mathbf{x}})=\frac{E_{S D}}{M_{S}} \sum_{j=1}^{N} \sum_{i=1}^{M_{S}}\left|h_{S D, j}^{i}\right|^{2} \lambda+N \lambda E_{S D} .
$$

It can be easily verified that (45) has a similar form to (29). Therefore, the PEP is found as

$$
\begin{aligned}
P(\mathbf{x}, \hat{\mathbf{x}})_{\text {end-to-end }} & \leq\left(\frac{E_{S D}}{4 M_{S} N_{0}}\right)^{-N M_{S}} \lambda^{-N M_{S}} \\
& \times \exp \left(-N \lambda \frac{E_{S D}}{4 N_{0}}\right) .
\end{aligned}
$$

We observe from (46) that the exponential term dominates and provides an AWGN-like performance similar to our observation for CSI-assisted AaF relaying under non-fading $R \rightarrow D$ link assumption.

For the convenience of the reader, Table I tabulates the diversity orders of CSI-assisted $\mathrm{AaF}$, blind $\mathrm{AaF}$ and $\mathrm{DaF}$ in the all aforementioned scenarios.

\section{Simulation Results AND Discussion}

In this section, we present Monte-Carlo simulation results for cooperative transmission systems which have been

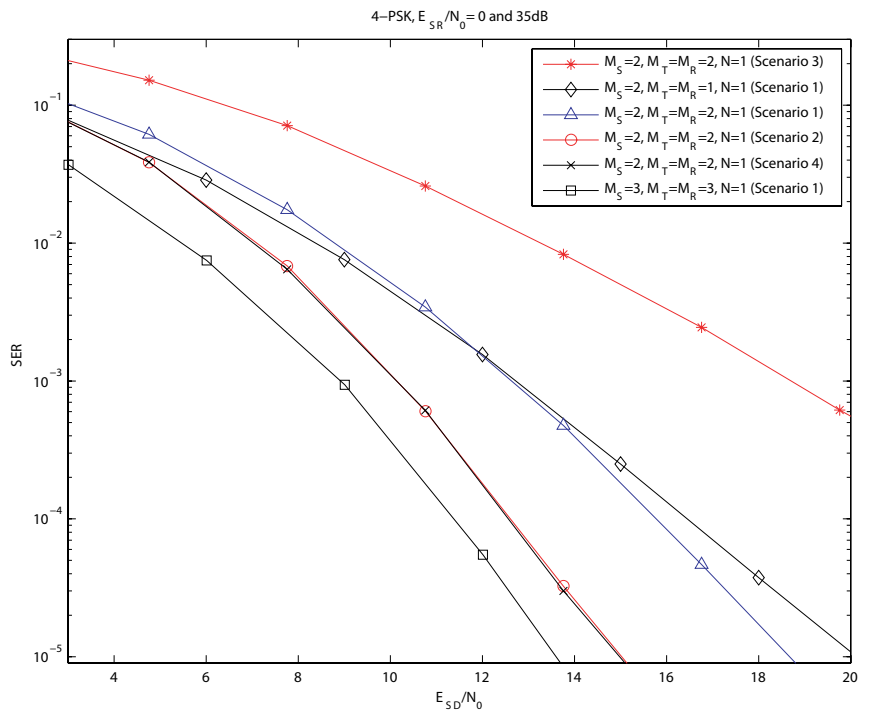

Fig. 2. SER performance of blind AaF relaying.

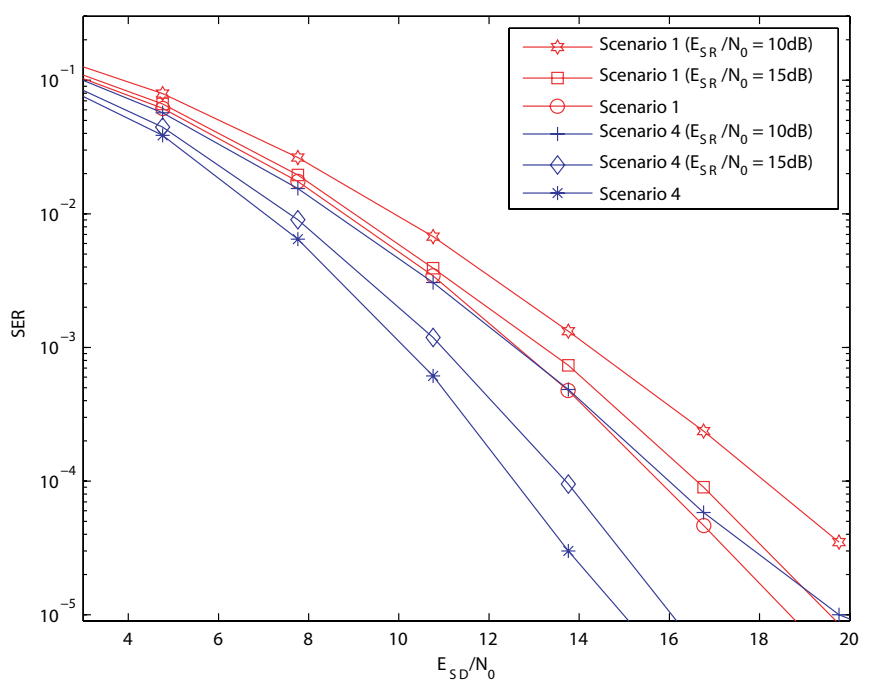

Fig. 3. SER performance of blind AaF relaying assuming $M=2$.

described and analyzed in this paper. We employ 4-PSK modulation and consider the aforementioned scenarios with the following numerical values:

- Scenario 1: $E_{S R} / N_{0}=35 \mathrm{~dB}$ (unless otherwise indicated) and $E_{S D}=E_{R D}$.

- Scenario 2: $E_{R D} / N_{0}=35 \mathrm{~dB}$ and $E_{S D}=E_{S R}$.

- Scenario 3: $E_{S R} / N_{0}=0 \mathrm{~dB}$ and $E_{S D}=E_{R D}$.

- Scenario 4: $E_{S R} / N_{0}=35 \mathrm{~dB}$ (unless otherwise indicated), $E_{S D}=E_{R D}$, and non-fading $R \rightarrow D$.

In Fig. 2, we illustrate the SER (symbol error rate) performance of the blind AaF scheme assuming $M_{S}=M_{R}=$ $M_{T}=M=2$ and $M_{S}=M_{R}=M_{T}=M=3$ with $N=1$. For $M=2$ and 3 , we consider Alamouti scheme and G3-STBC [Eq. (37) of 2], respectively, as the underlying space-time codes. For Scenario 1 where $S \rightarrow D$ and $R \rightarrow D$ links are balanced, it is observed that the diversity orders for $(M=2, N=1)$ and $(M=3$, $N=1)$ are 4 and 6 , respectively, confirming the diversity order of $M_{T} \min \left(M_{S}, N\right)+M_{S} N$ observed through our PEP 


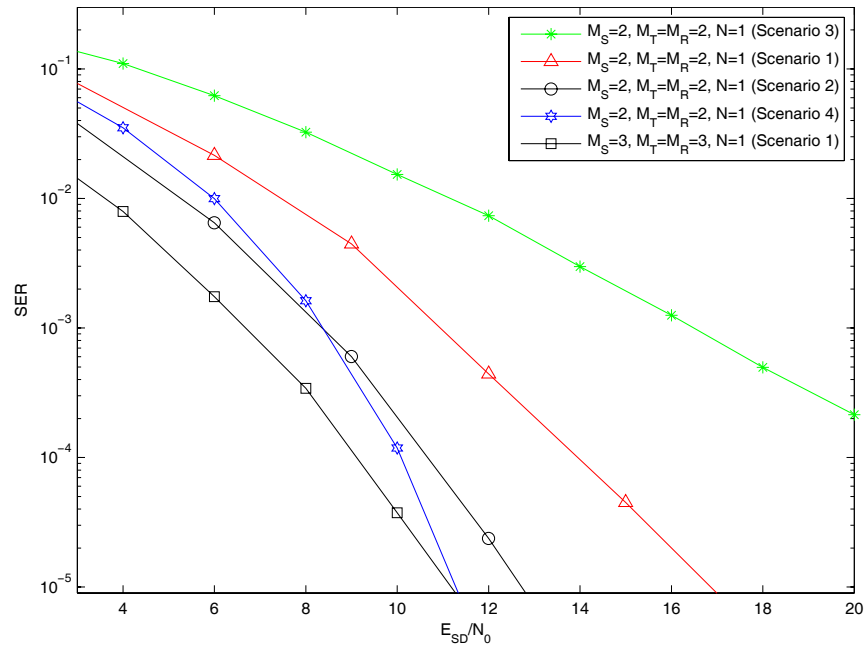

Fig. 4. SER performance of CSI-assisted AaF relaying.

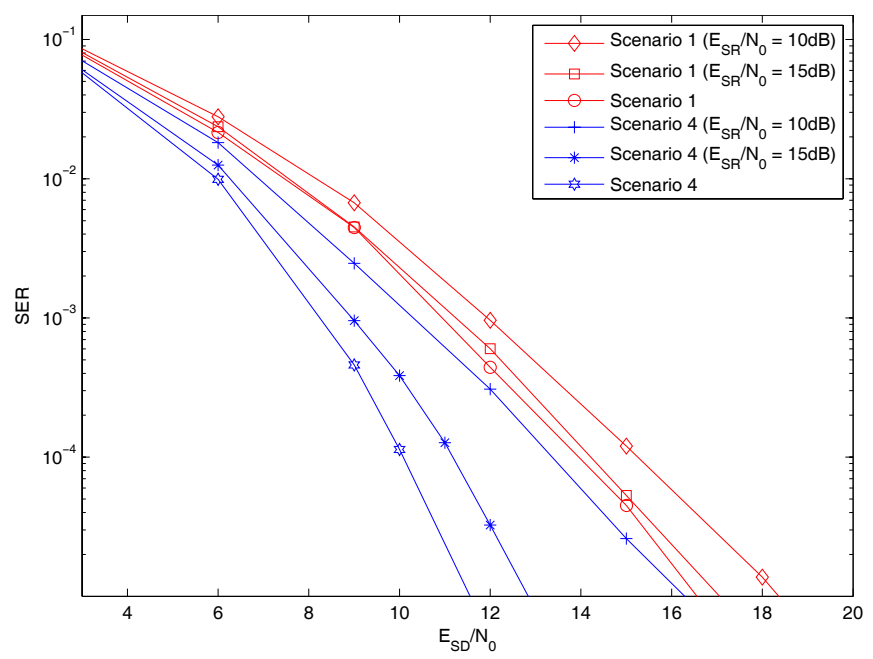

Fig. 5. SER performance of CSI-assisted AaF relaying assuming $M=2$.

expressions in (32)-(34). To further confirm our diversity order analysis, we also include the performance for $M_{R}=M_{T}=1$ while keeping $M_{S}=1$. It is clearly seen that the achievable diversity order in this case is 3 . For Scenario 2 where $S \rightarrow R$ and $S \rightarrow D$ links are balanced, it is observed that the diversity order is 6 for $(M=2, N=1)$, confirming our PEP derivation of (36). For poor inter-user channel (Scenario 3), the diversity order of blind $\mathrm{AaF}$ scheme is limited to $M_{S} N$, i.e., the diversity order of non-cooperative transmission confirming our observation in (37). On the other hand, in the presence of nonfading $R \rightarrow D$ link with $M=2$ and $N=1$, it is observed that the diversity order is 6 confirming our observation in (39) for Scenario 4. In Fig. 3, we investigate the performance of blind AaF scheme assuming moderate SNR values in the $S \rightarrow R$ link. Specifically, we consider Scenarios 1 and 4 under the assumption of $E_{S R} / N_{0}=10 \mathrm{~dB}$ and $15 \mathrm{~dB}$. For comparison purpose, we include the performance plots associated with $E_{S R} / N_{0}=35 \mathrm{~dB}$. It is observed from Fig. 3 that the slopes of performance curves (i.e., diversity order) are still preserved for $E_{S R} / N_{0}=15 \mathrm{~dB}$. However, loss in diversity order is observed when $E_{S R} / N_{0}$ reduces to $10 \mathrm{~dB}$.

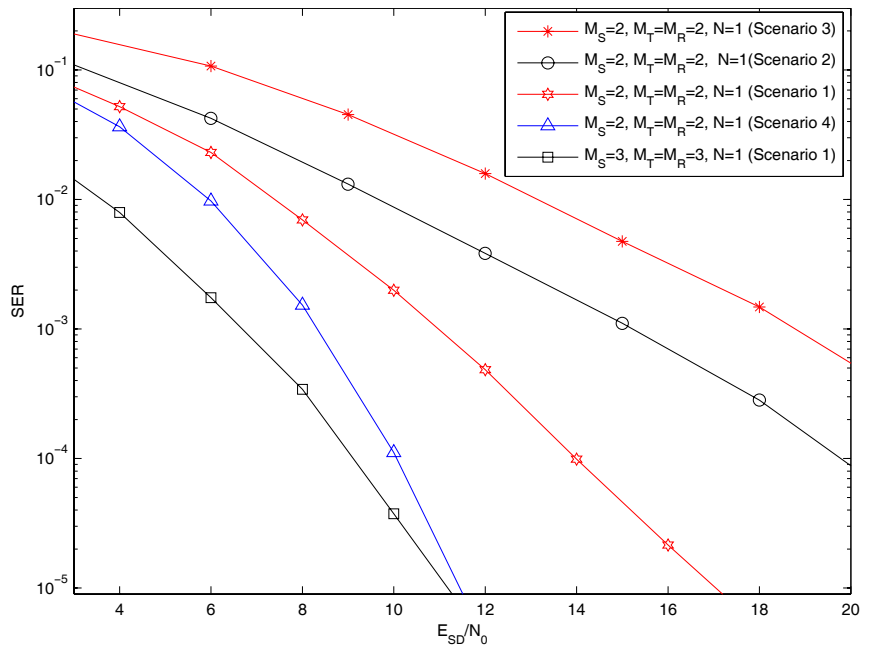

Fig. 6. SER performance of DaF relaying.

In Fig. 4, we illustrate the SER performance of the CSIassisted AaF relaying scheme. For Scenario 1 with $(M=2$, $N=1)$ and $(M=3, N=1)$, we observe diversity orders of 4 and 6 , respectively, confirming the diversity order of $N\left(M_{T}+\right.$ $\left.M_{S}\right)$ observed from our PEP expression in (25). For Scenario 2 with $(M=2, N=1)$ the diversity order is 6 , confirming our observation in (27). For Scenario 4 with the non-fading $R \rightarrow D$ link, we observe a rapid steep which is expected due to the presence of the exponential term in (30). For Scenario 3 , the diversity order remains limited to the diversity order of non-cooperative transmission, confirming our observation in (28). In Fig. 5, we investigate the performance of CSI-assisted $\mathrm{AaF}$ scheme assuming moderate SNR values in $S \rightarrow R$ link. Under the assumption of $E_{S R} / N_{0}=15 \mathrm{~dB}$, full diversity order is observed for Scenarios 1 and 4 . On the other hand, diversity order loss is observed for $E_{S R} / N_{0}=10 \mathrm{~dB}$ similar to the earlier observation for the blind scheme.

In Fig. 6, we present the SER performance of the $\mathrm{DaF}$ relaying scheme. Comparison of Figs. 4 and 6 for Scenario 1 reveals that $\mathrm{DaF}$ and $\mathrm{CSI}$-assisted $\mathrm{AaF}$ protocols yield identical performance, confirming our observations in (25), (30), (43), and (46). For $E_{S R} / N_{0}=0 \mathrm{~dB}$ (Scenario 3), both $\mathrm{DaF}$ and CSI-assisted $\mathrm{AaF}$ achieve the same diversity order, but the latter outperforms due to additional coding gain. For Scenario 2 , DaF relaying suffers from relay's assumed location under the considered scenario. Its performance is limited to the noncooperative case and is therefore significantly outperformed by CSI-assisted $\mathrm{AaF}$ relaying.

In Fig. 7, we present the SER performance of DaF scheme for moderate $S N R$ values in $S \rightarrow R$ link, i.e., $E_{S R} / N_{0}=10$ and $15 \mathrm{~dB}$. For $E_{S R} / N_{0}=15 \mathrm{~dB}$, the performance degradation with respect to $E_{S R} / N_{0}=35 \mathrm{~dB}$ is small. However, for $E_{S R} / N_{0}=10 \mathrm{~dB}$, the performance is severely degraded and a loss in diversity order is observed as $\mathrm{DaF}$ scheme suffers from error propagation.

\section{CONCLUSion}

In this paper, we have investigated performance of three relaying schemes in a cooperative scenario in which the cooperating nodes are equipped with multiple antennas and 


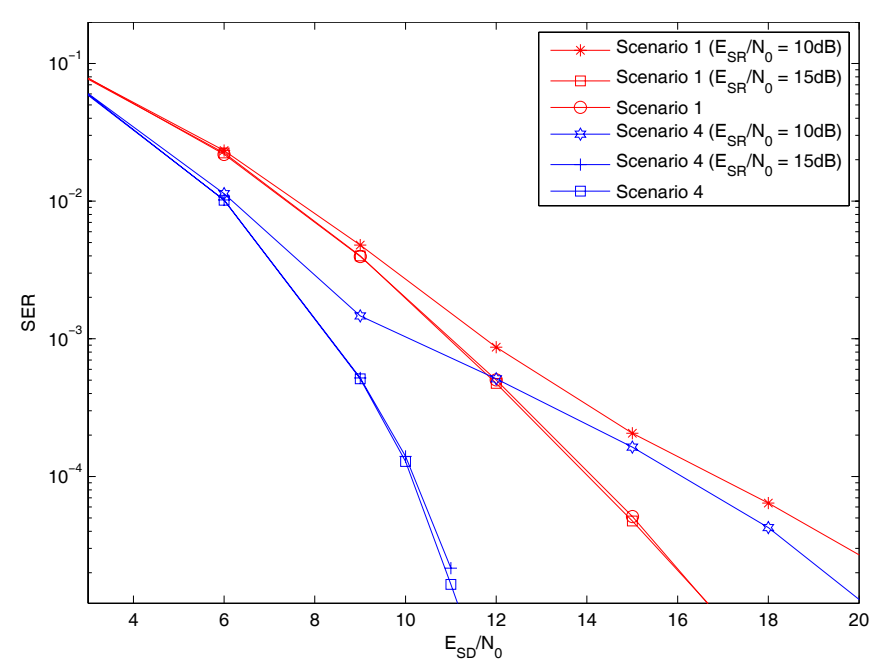

Fig. 7. SER performance of DaF relaying assuming $M=2$.

operating over frequency-flat Rayleigh fading channels. In particular, we have analyzed the diversity gains of blind $\mathrm{AaF}$, CSI-assisted $\mathrm{AaF}$, and $\mathrm{DaF}$ schemes in a single relay assisted transmission scenario where the source, relay, and destination terminals are equipped with $M_{S}, M_{T}=M_{R}$, and $N$ antennas, respectively. Under the assumption of balanced $S \rightarrow D$ and $R \rightarrow D$ links and sufficiently large SNR for the $S \rightarrow R$ link, we have demonstrated that the maximum achievable diversity order is $M_{T} \min \left(M_{S}, N\right)+M_{S} N$ for the blind $\mathrm{AaF}$ scheme and $N\left(M_{S}+M_{T}\right)$ for both CSIassisted $\mathrm{AaF}$ and $\mathrm{DaF}$ schemes. For another scenario where $S \rightarrow R$ and $S \rightarrow D$ links are balanced and sufficiently large SNR for the $R \rightarrow D$ link, we have shown that blind AaF scheme achieves a diversity order of $M_{S}\left(N+M_{T}\right)$ while the diversity orders for CSI-assisted $\mathrm{AaF}$ and $\mathrm{DaF}$ schemes are given by $M_{S}\left(N+M_{R}\right)$ and $M_{S} N$, respectively. We have also considered other scenarios involving the availability of a non-fading $R \rightarrow D$ link and poor inter-user channel quality. We have further presented a comprehensive Monte Carlo simulation study to corroborate the analytical results and to provide detailed performance comparisons among the three relaying techniques under various scenarios.

\section{APPENDIX A}

DERIVATION OF (32)-(33)-(34)

In this Appendix, we derive PEP expressions for blind $\mathrm{AaF}$ scheme assuming $E_{R D} / N_{0}=E_{S D} / N_{0}$ and $E_{S R} / N_{0}>>$ $E_{S D} / N 0$. Under these assumptions, $\gamma^{t}$ in (19) can be approximated as $\gamma^{t} \approx 1 / M_{T}$. Thus, $d^{2}(\mathbf{x}, \hat{\mathbf{x}})$ in (31) reduces to

$$
\begin{array}{r}
d^{2}(\mathbf{x}, \hat{\mathbf{x}})=\frac{\lambda E_{S D}}{M_{T}} \sum_{t=1}^{M_{T}} \sum_{j=1}^{N}\left|h_{R D, j}^{t}\right|^{2} \sum_{i=1}^{M_{S}}\left|h_{S R, t}^{i}\right|^{2} \\
+\frac{\lambda E_{S D}}{M_{S}} \sum_{j=1}^{N} \sum_{i=1}^{M_{S}}\left|h_{S D, j}^{i}\right|^{2} .
\end{array}
$$

Due to high SNR assumption in the $S \rightarrow R$ link, we assume that the correlation of noise terms at different receive antennas of the destination terminal is negligible. Introducing
$Y_{1}=\sum_{j=1}^{N} \sum_{i=1}^{M_{S}}\left|h_{S D, j}^{i}\right|^{2}$ and $Y_{2}^{t}=Z_{1}^{t} Z_{2}^{t}$ with $Z_{1}^{t}=$ $\sum_{j=1}^{N}\left|h_{R D, j}^{t}\right|^{2}$ and $Z_{2}^{t}=\sum_{i=1}^{M_{S}}\left|h_{S R, t}^{i}\right|^{2}$, we can rewrite (47) as

$$
d^{2}(\mathbf{x}, \hat{\mathbf{x}})=\frac{\lambda E_{S D}}{M_{S}} Y_{1}+\frac{\lambda E_{S D}}{M_{T}} \sum_{t=1}^{M_{T}} Y_{2}^{t} .
$$

After substituting (48) in (22), we have the PEP expression as

$$
\begin{array}{r}
P\left(\mathbf{x}, \hat{\mathbf{x}} \mid Y_{1}, Y_{2}\right) \leq \exp \left(-\frac{\lambda E_{S D}}{4 N_{0} M_{S}} Y_{1}\right) \\
\times \prod_{t=1}^{M_{T}} \exp \left(-\frac{\lambda E_{S D}}{4 N_{0} M_{T}} Y_{2}^{t}\right) .
\end{array}
$$

The unconditional PEP can be obtained as [18]

$$
P(\mathbf{x}, \hat{\mathbf{x}}) \leq\left.\left.\Phi_{Y_{1}}(\omega)\right|_{j \omega=-\frac{\lambda E_{S D}}{4 M_{S} N_{0}}} \prod_{t=1}^{M_{T}} \Phi_{Y_{2}^{t}}(\omega)\right|_{j \omega=-\frac{\lambda E_{S} P}{4 M_{T} \mathbb{N}_{0}}},
$$

where $\Phi_{Y_{1}}(\omega)$ and $\Phi_{Y_{2}^{t}}(\omega)$ are the characteristic functions of $Y_{1}$ and $Y_{2}^{t}$, respectively. Since $\left|h_{S D, j}^{i}\right|$ is Rayleigh distributed, the characteristic function of $Y_{1}$ can be readily found as

$$
\left.\Phi_{Y_{1}}(\omega)\right|_{j \omega=-\frac{\lambda E_{S D}}{4 M_{S} N_{0}}}=\left(1+\frac{E_{S D}}{4 M_{S} N_{0}} \lambda\right)^{-M_{S} N},
$$

where the first term can be further ignored under high SNR assumption. In the following, we will derive the PEP expression for three cases:

Case $1\left(M_{S}<N\right)$ : The characteristic function of $Y_{2}$ can be evaluated as [19]

$$
\Phi_{Y_{2}^{t}}(\omega)=\int_{0}^{\infty} f_{Z_{1}^{t}}\left(z_{1}^{t}\right) \Phi_{Z_{2}^{t}}\left(\omega z_{1}^{t}\right) d z_{1}^{t},
$$

where $f_{Z_{1}^{t}}\left(z_{1}^{t}\right)$ is the probability density function (pdf) of $Z_{1}^{t}$ and $\Phi_{Z_{2}^{t}}\left(\omega z_{1}^{t}\right)$ has the similar form as in (51). Here, $Z_{1}^{t}$ is a chi-squared random variable with $2 N$ degrees of freedom with the pdf $f_{Z_{1}^{t}}\left(z_{1}^{t}\right)=z_{1}^{t^{N-1}} e^{-z_{1}^{t}} / \Gamma(N)$ [20] where $\Gamma($.) denotes the gamma function [21]. This leads to

$$
\begin{aligned}
\left.\Phi_{Y_{2}^{t}}(\omega)\right|_{j \omega=} & =\frac{\lambda E_{S D}}{4 M_{T} N_{0}}=\frac{1}{\Gamma(N)}\left(\frac{E_{S D}}{4 M_{T} N_{0}}\right)^{-M_{S}} \lambda^{-M_{S}} \\
& \times \int_{0}^{\infty}\left(\frac{4 M_{T} N_{0}}{\lambda E_{S D}}+z_{1}^{t}\right)^{-M_{S}} z_{1}^{t^{N-1}} e^{-z_{1}^{t}} d z_{1}^{t}
\end{aligned}
$$

Assuming $E_{S D} / N_{0}>>1$ and using the integral form given by [21], we obtain

$$
\begin{aligned}
\left.\Phi_{Y_{2}^{t}}(\omega)\right|_{j \omega}=-\frac{\lambda E_{S D}}{4 M_{T} N_{0}} & =\frac{\Gamma\left(N-M_{S}\right)}{\Gamma(N)} \\
& \times\left(\frac{E_{S D}}{4 M_{T} N_{0}}\right)^{-M_{S}} \lambda^{-M_{S}} .
\end{aligned}
$$

Substituting (54) and (51) in (50), we find the final PEP expression as given in (32).

Case $2\left(M_{S}>N\right)$ : Noting that this case is similar to the previous case with $N$ and $M_{S}$ now interchanged, we simply follow similar steps and find the PEP expression as given in (33).

Case $3\left(M_{S}=N\right)$ : Following the same argument in Case 1 
and further defining $u=v+z_{1}^{t}$ where $v=4 M_{S} N_{0} /\left(\lambda E_{S D}\right)$, we write (53) as

$$
\begin{array}{r}
\left.\Phi_{Y_{2}^{t}}(\omega)\right|_{j \omega=-\frac{\lambda E_{S D}}{4 M_{T} N_{0}}}=\frac{1}{\Gamma(N)}\left(\frac{E_{S D}}{4 M_{T} N_{0}}\right)^{-M_{S}} \lambda^{-M_{S}} \\
\times \exp (v) \int_{v}^{\infty} u^{-1}(1-v / u)^{N-1} e^{-u} d u .
\end{array}
$$

Under $E_{S D} / N_{0}>>1$ assumption, we have $1-v / u \approx 1$. Hence, we can rewrite (55) as

$$
\begin{array}{r}
\left.\Phi_{Y_{2}^{t}}(\omega)\right|_{j \omega=-\frac{\lambda E_{S D}}{4 M_{T} N_{0}}}=\frac{1}{\Gamma(N)}\left(\frac{E_{S D}}{4 M_{T} N_{0}}\right)^{-M_{S}} \lambda^{-M_{S}} \\
\quad \times \exp (v) \Gamma(0, v),
\end{array}
$$

where $\Gamma(a, b)=\int_{b}^{\infty} q^{a-1} \exp (-q) \mathrm{d} q[21]$ denotes the incomplete gamma function. Using the limiting approximation $\Gamma(0, v) \approx-\log (v)$ for $v \rightarrow 0$ [21], (56) reduces to

$$
\begin{array}{r}
\left.\Phi_{Y_{2}^{t}}(\omega)\right|_{j \omega=-\frac{\lambda E_{S D}}{4 M_{T} N_{0}}}=\frac{1}{\Gamma(N)}\left(\frac{E_{S D}}{4 M_{T} N_{0}}\right)^{-M_{S}} \lambda^{-M_{S}} \\
\times \log \left(\frac{E_{S D}}{N_{0}}\right) .
\end{array}
$$

Substituting (57) and (51) in (50), we find the final PEP expression as given in (34).

\section{REFERENCES}

[1] V. Tarokh, N. Seshadri, and A. Calderbank, "Space-time codes for high data rate wireless communication: performance criterion and code construction," IEEE Trans. Inform. Theory, vol. 44, no. 2, pp. 744-765, 1998.

[2] V. Tarokh, H. Jafarkhani, and A. Calderbank, "Space-time block codes from orthogonal designs," IEEE Trans. Inform. Theory, vol. 45, no. 5, pp. 1456-1467, 1999.

[3] S. Alamouti, "A simple transmit diversity technique for wireless communications," IEEE J. Select. Areas Commun., vol. 16, no. 8, pp. 14511458,1998

[4] A. Sendonaris, E. Erkip, and B. Aazhang, "User cooperation diversitypart I: system description," IEEE Trans. Commun., vol. 51, no. 11, pp. 1927-1938, 2003.

[5] — - "User cooperation diversity-part II: implementation aspects and performance analysis," IEEE Trans. Commun., vol. 51, no. 11, pp. 19391948, 2003.

[6] J. Laneman, D. Tse, and G. Wornell, "Cooperative diversity in wireless networks: efficient protocols and outage behavior," IEEE Trans. Inform. Theory, vol. 50, no. 12, pp. 3062-3080, 2004.

[7] M. Janani, A. Hedayat, T. Hunter, and A. Nosratinia, "Coded cooperation in wireless communications: space-time transmission and iterative decoding," IEEE Trans. Signal Processing, vol. 52, no. 2, pp. 362-371, 2004.

[8] J. Laneman and G. Wornell, "Distributed space-time-coded protocols for exploiting cooperative diversity in wireless networks," IEEE Trans. Inform. Theory, vol. 49, no. 10, pp. 2415-2425, 2003.

[9] M. Hasna and M. Alouini, "A performance study of dual-hop transmissions with fixed gain relays," IEEE Trans. Wireless Commun., vol. 3, no. 6, pp. 1963-1968, 2004.

[10] R. Nabar, H. Bolcskei, and F. Kneubuhler, "Fading relay channels: performance limits and space-time signal design," IEEE J. Select. Areas Commun., vol. 22, no. 6, pp. 1099-1109, 2004.

[11] K. Azarian, H. Gamal, and P. Schniter, "On the achievable diversitymultiplexing tradeoff in half-duplex cooperative channels," IEEE Trans. Inform. Theory, vol. 51, no. 12, pp. 4152-4172, 2005.
[12] A. Adinoyi and H. Yanikomeroglu, "Cooperative relaying in multiantenna fixed relay networks," IEEE Trans. Wireless Commun., vol. 6 , no. 2, pp. 533-544, 2007.

[13] B. Wang, J. Zhang, and A. Host-Madsen, "On the capacity of MIMO relay channels," IEEE Trans. Inform. Theory, vol. 51, no. 1, pp. 29-43, 2005.

[14] S. Yiu, R. Schober, and L. Lampe, "Distributed space-time block coding for cooperative networks with multiple-antenna nodes," in Proc. IEEE Workshop on Computational Advances in Multi-Sensor Adaptive Processing (CAMSAP), Puerto Vallarta, Mexico, Dec. 2005, pp. 52-55.

[15] — , "Distributed space-time block coding," IEEE Trans. Commun., vol. 54, no. 7, pp. 1195-1206, 2006.

[16] H. Mheidat and M. Uysal, "Impact of receive diversity on the performance of amplify-and-forward relaying under APS and IPS power constraints," IEEE Commun. Lett., vol. 10, no. 6, pp. 468-470, 2006.

[17] Y. Jing and B. Hassibi, "Cooperative diversity in wireless relay networks with multiple-antenna nodes," in Proc. IEEE International Symposium on Information Theory, Sept. 2005, pp. 815-819.

[18] M. K. Simon and M. S. Alouini, Digital Communication Over Fading Channels: A Unified Approach to Performance Analysis. New York: Wiley-Interscience, 2000.

[19] A. Stuart and J. K. Ord, Kendall's Advanced Theory of Statistics. Griffin and Co., 1987.

[20] J. Proakis, Digital Communications, 2nd ed. New York: McGraw-Hill, 1989.

[21] I. S. Gradshteyn and I. M. Ryzhik, Table of Integrals, Series and Products. Academic Press, 2000

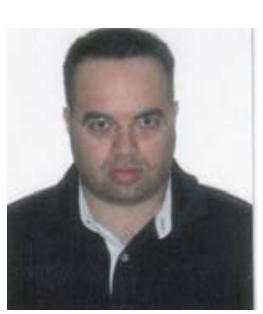

Hakam Muhaidat received the B.Sc. in Communication Engineering from Yarmouk University, Irbid, Jordan in 1995, M.Sc. in Electrical Engineering from University of Wisconsin, Milwaukee, USA in 1999, and the Ph.D. degree in Electrical Engineering from University of Waterloo, Waterloo, Ontario, in 2006. From 1997 to 1999 , he worked as a Research and Teaching Assistant in the Signal Processing Group at the University of Wisconsin. From 2006 to 2008 , he was a postdoctoral fellow in the Department of Electrical and Computer Engineering, University of Toronto, Canada. He is currently an Assistant Professor with the School of Engineering Science at Simon Fraser University, Burnaby, Canada.

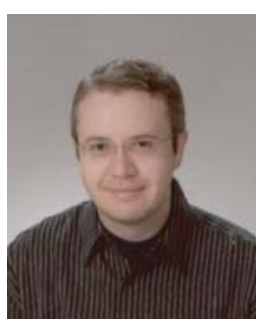

Murat Uysal was born in Istanbul, Turkey in 1973. $\mathrm{He}$ received the B.Sc. and the M.Sc. degree in electronics and communication engineering from Istanbul Technical University, Istanbul, Turkey, in 1995 and 1998, respectively, and the Ph.D. degree in electrical engineering from Texas A\&M University, College Station, Texas, in 2001. Since 2002, he has been with the Department of Electrical and Computer Engineering, University of Waterloo, Canada, where he is now an Associate Professor. His general research interests lie in communications theory and signal processing for communications with special emphasis on wireless applications. Specific research areas include space-time coding, MIMO techniques, cooperative communications, performance analysis over fading channels, and free-space optical communications.

Dr. Uysal is an Associate Editor for IEEE TRANSACTIONS ON WIRELESS COMMUNICATIONS and IEEE COMMUNICATIONS LETTERS. He also served as a Guest Co-Editor for WILEY JOURNAL ON WiRELESS COMMUNICATIONS AND Mobile Computing's Special Issue on "MIMO Communications" published in 2004. Over the years, he has served on the technical program committee of more than 50 international conferences in the communications area. He co-chaired IEEE ICC (International Conference on Communications) Communication Theory Symposium in 2007 and chaired IEEE CCECE (Canadian Conference on Electrical and Computer Engineering) Communications and Networking Symposium in 2008. Dr. Uysal is a Senior IEEE member. 